\title{
An Optimality Criterion for the Crossing Number
}

\author{
László A. Székely † \\ Department of Mathematics \\ University of South Carolina, Columbia, SC 29208, USA
}

Received 30 September 2007, accepted 22 February 2008, published online 19 June 2008

\begin{abstract}
A sufficient condition is given that a certain drawing minimizes the crossing number. The condition is in terms of intersections in an arbitrary set system related to the drawing, and is like a correlation inequality.
\end{abstract}

Keywords: Graph drawing, crossing number.

Math. Subj. Class.: 05C10, 05C62

\section{Definitions}

First I recall from [5] definitions for two kinds of drawings, in which different kinds of crossing numbers can be conveniently set.

A drawing $D$ of a finite graph $G$ on the plane is an injection $\phi$ from the vertex set $V(G)$ into the plane, and a mapping of the edge set $E(G)$ into the set of simple plane curves, i.e. homeomorphic images of the interval $[0,1]$, such that the curve corresponding to the edge $e=u v$ has endpoints $\phi(u)$ and $\phi(v)$, and contains no more points from the image of $\phi$.

We say that two edges in a drawing cross in a certain point of the plane, or the point is a crossing point of the two edges, if this point belongs to the interiors of the curves representing the edges. The number of crossings $\operatorname{cr}(D)$ in the drawing $D$ is the sum of the number of crossing points for all unordered pairs of edges.

A drawing $D$ is normal if it satisfies (i), (ii) and (iii):

(i) any two of the curves have finitely many points in common; and

(ii) no two curves have a point in common in a tangential (touching) way

(i.e. defining locally the "left side" and the "right side" of the curves at the common point, both curves are present at both sides of each in every small neighborhood of that point);

\footnotetext{
${ }^{\dagger}$ Research partially supported by the NSF Grants 0302307 and 0701111 .

E-mail address: szekely@math.sc.edu (László A. Székely)
} 
(iii) no point of the plane belongs to the interior of three curves, each representing an edge of the graph.

A drawing $D$ is nice, if it is normal, and in addition satisfies

(iv) no two adjacent edges (i.e. edges sharing an endpoint) cross; and

(v) any two edges cross at most once.

The crossing number $C R(G)$ of the graph $G$ is the minimum of $\operatorname{cr}(D)$ over all drawings of $G$. We call a drawing $D$ optimal (for $C R$ ) if it realizes $\operatorname{cr}(D)=C R(G)$ ). It is easy to see that an optimal drawing must satisfy (i) and (ii), and a little work shows that it also must satisfy (iv) and (v). (Condition (iii) makes little difference other than allowing a simpler definition of $C R(G)$ by counting crossings in $D$, instead of looking at pairs of edges.) Therefore, we have an equivalent definition of $C R(G)$ : the minimum of $\operatorname{cr}(D)$ over all normal, nice drawings of $G$.

Pach and Tóth [3] introduced two new variants of the crossing number problem: the pairwise crossing number $C R-P A I R(G)$ is equal to the minimum number of unordered pairs of edges that cross each other at least once (i.e. they are counted once instead of as many times they cross), over all normal drawings of $G$; and

the odd crossing number $C R-O D D(G)$ is equal to the minimum number of unordered pairs of edges that cross each other odd times, over all normal drawings of $G$.

In Tutte's work [8] another kind of crossing number is implicit:

the independent-odd crossing number. Let $c r$-iodd $(D)$ denote the number of unordered pairs of non-adjacent edges that cross each other odd times in a normal drawing $D$ of the graph $G$, and let $C R-I O D D(G)$ denote the minimum of $c r$-iodd $(D)$ over all normal drawings $D$ of G.

The following chain of inequalities is obvious from the definitions:

$$
C R-I O D D(G) \leq C R-O D D(G) \leq C R-P A I R(G) \leq C R(G) .
$$

No example of strict inequality was known for a long time, but in a recent work Pelsmajer, Schaefer and Štefankovič [4] showed examples of graphs $G$ with $C R-O D D(G)<C R(G)$.

\section{The criterion}

Let us associate with every edge $e=\{x, y\} \in E(G)$ an arbitrary vertex set $A_{e} \subseteq V(G) \backslash$ $\{x, y\}$. If the edges $e$ and $f$ are non-adjacent, then we define the parity of this edge pair as 0 or 1 according to

$$
\operatorname{par}(e, f)=\left|e \cap A_{f}\right|+\left|f \cap A_{e}\right| \text { modulo } 2 .
$$

Let us be given a normal drawing $D$ of the simple graph $G$. If non-adjacent edges $e, f$ cross in $D$ odd times, then we write $e \times{ }_{D}^{o d d} f$, otherwise write $e \times{ }_{D}^{e v e n} f$. Later, if not specified otherwise, single summations written for pairs of edges mean summations are for unordered pairs of non-adjacent edges.

Theorem 1. Using the notation above, the condition that

$$
\text { for all } e \mapsto A_{e} \text { assignments } \sum_{\substack{p a r(e, f)=1 \\ e \times d_{D} d_{f}}} 1 \leq \sum_{\substack{p a r(e, f)=1 \\ e \times e^{e} e_{f}}} 1
$$

holds, is equivalent to the CR-IODD-optimality of $D$. Furthermore, if $D$ is nice, then $D$ is optimal for $C R$ as well. 
It is hard to verify whether this criterion holds, since checking the condition for all possible sets $A_{e}$ requires exponential time. This not surprising, in view of the NP-completeness of the decision problems $C R(G) \leq k$ (Garey and Johnson [1]), of $C R-O D D(G) \leq k$ (Pach and Tóth [3]), and the NP-hardness of the decision problem $C R-P A I R(G) \leq k$ (Pach and Tóth [3]). However, inequality (3), which looks like a correlation inequality formulated about random edge pairs, may have a less exhaustive and more theoretical proof for some graphs and their drawings that show a high degree of structure. A natural candidate would be Zarankiewicz' drawing of the complete bipartite graph, which is nice and conjectured to be optimal. For the fascinating history of Turán's Brick Factory Problem [7] and the Zarankiewicz drawing [10], see [6] and the classic [2]. For the best current result, see [9].

Note that if the second part of the criterion applies as the drawing is nice, then $C R(G)$ equals to $C R-I O D D(G)$.

\section{Proof of the criterion}

We recall what we need from [5]. Let us be given an arbitrary cyclic order $\mathcal{C}=v_{1}, v_{2}, \ldots, v_{n}$ of the vertices of a simple graph $G$. We say that two non-adjacent edges of $G$, say $x y$ and $u z$ are in acyclic order, if the cyclic order $\mathcal{C}$ restricted to these 4 vertices is $x, u, y, z$ or $x, z, y, u$. Otherwise, two non-adjacent edges are in cyclic order. These two relations are clearly symmetric. In [5] we defined the relation $O_{\mathcal{C}}$ of non-adjacent edges of $G$ as follows:

$$
O_{\mathcal{C}}(x y, u z)= \begin{cases}1 & \text { if } x y \text { and } u z \text { are in cyclic order } \\ 0 & \text { otherwise }\end{cases}
$$

Note that $O_{\mathcal{C}}(x y, u z)=O_{\mathcal{C}}(x y, z u)=O_{\mathcal{C}}(u z, x y)$.

Let us be given a normal drawing $D$ of the graph $G$ and a fixed $O_{\mathcal{C}}$. In [5] we associated with the curve representing the edge $e=\{x, y\} \in E(G)$ a particular function $Q_{x y}: V(G) \backslash$ $\{x, y\} \rightarrow\{1,-1\}$, which also depends on the binary relation $O_{\mathcal{C}}$, such that changing any function $Q_{x y}$ to $-Q_{x y}$ makes no difference; and we also observed in [5] that any set of functions

$$
\mathcal{Q}=\left\{Q_{a b}: V(G) \backslash\{a, b\} \rightarrow\{1,-1\}: a b \in E(G)\right\}
$$

comes from some normal drawing through this association.

(Though just citing (6) would suffice to prove our theorem without even giving the definition of $Q_{x y}$ here, for the geometrically inclined Reader, we sketch the definition of $Q_{x y}$. Deform the normal drawing $D$ without pulling edges over vertices and keeping normality such that the vertex set of $G$ lies on the unit circle around the origin in cyclic order $\mathcal{C}$. Consider the following - possibly self-intersecting — closed curve $q$ in the plane extended with the point $\infty$ : the ray from $x$ to $\infty$ not passing through the origin, the ray from $\infty$ to $y$ not passing through the origin, and the curve representing the $y x$ edge in the deformed drawing. It is shown in [5] that this closed curve defines two classes of vertices of $V(G) \backslash\{x, y\}$ (one of them can be empty) such that a generic curve connecting $u, v \in V(G) \backslash\{x, y\}$ passes through $q$ odd number of times if and only if $u$ and $v$ belong to different classes. $Q_{x y}$ takes value 1 on the elements of one equivalence class, and takes value -1 on the elements of the other equivalence class.) 
Formula (20) in [5] shows that $x y \times \underset{D}{o d d} u z$ holds if and only if

$$
\begin{aligned}
& {\left[1-O_{\mathcal{C}}(x y, u z)\right] \frac{1+Q_{x y}(u) Q_{x y}(z) Q_{u z}(x) Q_{u z}(y)}{2} } \\
+ & O_{\mathcal{C}}(x y, u z) \frac{1-Q_{x y}(u) Q_{x y}(z) Q_{u z}(x) Q_{u z}(y)}{2}
\end{aligned}
$$

is equal to 1 , and $x y \times{ }_{D}^{\text {even }} u z$ holds if and only if (6) is 0 . We keep (6) in this ugly form that we will use in our calculations, but will elucidate (6) in (8). (There is a typo after (20) in [5], as the minus sign is missing in the formula $P_{x y}(u z)=-Q_{x y}(u) Q_{x y}(z)$, but this is irrelevant regarding the conclusions.) Let us introduce the abbreviation

$$
Q(x y, u z)=Q_{x y}(u) Q_{x y}(z) Q_{u z}(x) Q_{u z}(y) .
$$

Exploiting (6), we obtain

$$
\begin{aligned}
x y \times{ }_{D}^{\text {odd }} u z & \Leftrightarrow\left[O_{\mathcal{C}}(x y, u z)=0 \wedge Q(x y, u z)=1\right] \\
& \vee \quad\left[O_{\mathcal{C}}(x y, u z)=1 \wedge Q(x y, u z)=-1\right] ; \\
x y \times{ }_{D}^{\text {even }} u z & \Leftrightarrow\left[O_{\mathcal{C}}(x y, u z)=0 \wedge Q(x y, u z)=-1\right] \\
& \vee \quad\left[O_{\mathcal{C}}(x y, u z)=1 \wedge Q(x y, u z)=1\right] .
\end{aligned}
$$

Summing up (6) for all ordered pairs of non-adjacent edges, one obtains that the number of edge pairs crossing odd times in every normal drawing $D$ is

$$
\frac{N}{2}-\frac{1}{2} \sum_{x y \in E(G)} \sum_{\substack{u z \in E(G) \\\{u, z\} \cap\{x, y\}=\emptyset}}\left\{O_{\mathcal{C}}(x y, u z)-\frac{1}{2}\right\} Q_{x y}(u) Q_{x y}(z) Q_{u z}(x) Q_{u z}(y),
$$

where $N$ denotes the number of unordered pairs of non-adjacent edges in $G$. (Though there are many ways to define the binary relation $O_{\mathcal{C}}$, all of them yield formally the same formula (9), since $\mathcal{Q}$ in (9) depends on the choice of the relation $O_{\mathcal{C}}$.)

Let us fix the graph $G$, and the binary relation $O_{\mathcal{C}}$. Consider two normal drawings of $G$, an arbitrary $D^{\prime}$, and a $D$ optimal for $C R-I O D D$. Let $\mathcal{Q}$ and $\mathcal{Q}^{\prime}$ denote the set of functions associated with $D$ and $D^{\prime}$, respectively. According to (9), the optimality of $D^{\prime}$ is equivalent to the inequality

$$
\begin{aligned}
& \sum_{x y \in E(G)} \sum_{\substack{u z \in E(G) \\
\{u, z\} \cap\{x, y\}=\emptyset}}\left\{O_{\mathcal{C}}(x y, u z)-\frac{1}{2}\right\} Q_{x y}^{\prime}(u) Q_{x y}^{\prime}(z) Q_{u z}^{\prime}(x) Q_{u z}^{\prime}(y) \\
\leq & \sum_{x y \in E(G)} \sum_{\substack{u z \in E(G) \\
\{u, z\} \cap\{x, y\}=\emptyset}}\left\{O_{\mathcal{C}}(x y, u z)-\frac{1}{2}\right\} Q_{x y}(u) Q_{x y}(z) Q_{u z}(x) Q_{u z}(y) .
\end{aligned}
$$

For every $e=\{x, y\} \in E(G)$, set

$$
A_{\{x, y\}}=\left\{z \in V(G) \backslash\{x, y\}: Q_{\{x, y\}}(z) Q_{\{x, y\}}^{\prime}(z)=-1\right\} .
$$

Using the definition (2), it is not difficult to see that

$$
Q_{x y}^{\prime}(u) Q_{x y}^{\prime}(z) Q_{u z}^{\prime}(x) Q_{u z}^{\prime}(y)=Q_{x y}(u) Q_{x y}(z) Q_{u z}(x) Q_{u z}(y)(-1)^{p a r(x y, u z)} .
$$


Using (13) and the abbreviation (7), one can equivalently rewrite (10-11), based on a case analysis for $O_{\mathcal{C}}$, as

$$
\begin{aligned}
& \sum_{\substack{x y, u z \in E(G) \\
O_{\mathcal{C}}(x y, u z)=0}}\left\{1-(-1)^{\operatorname{par}(x y, u z)}\right\} Q(x y, u z) \\
\leq & \sum_{\substack{x y, u z \in E(G) \\
O_{\mathcal{C}}(x y, u z)=1}}\left\{1-(-1)^{\operatorname{par}(x y, u z)}\right\} Q(x y, u z) .
\end{aligned}
$$

At this point we claim the following identities for (14) and (15), which we put into a less formal summation:

$$
\begin{aligned}
& \sum_{\substack{\mathcal{C}(x y, u z)=0 \\
\operatorname{par}(x y, u z)=1}} Q(x y, u z)
\end{aligned}
$$

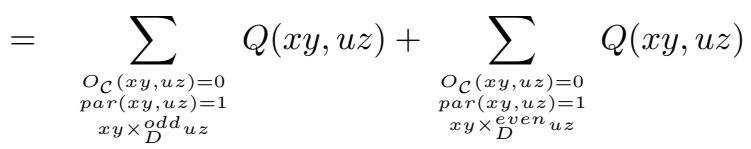

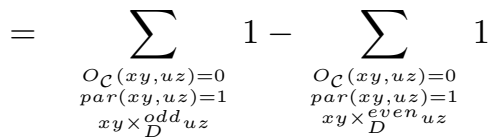

and

$$
\begin{aligned}
& \sum_{\substack{O_{\mathcal{C}}(x y, u z)=1 \\
\operatorname{par}(x y, u z)=1}} Q(x y, u z)
\end{aligned}
$$

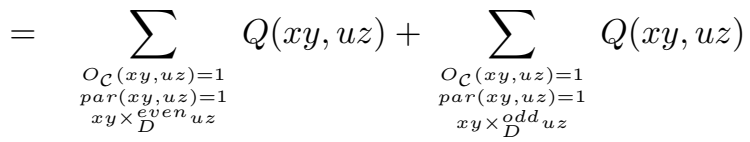

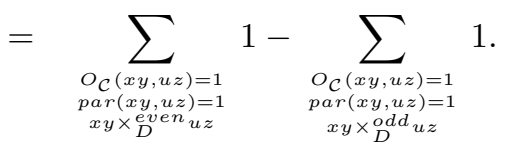

The equalities $(18)=(17)$, and $(20)=(21)$ follow from the fact, that the parity of the number of crossings of $x y$ and $u z$, together with the value of $O_{\mathcal{C}}(x y, u z)$ determines the value of $Q(x y, u z)$ as 1 or -1 , as substituted. This follows from (8). Finally, rewrite the inequality $(14) \leq$ (15) into the equivalent form $(18) \leq(21)$. Move the negative terms to the other side in the inequality $(18) \leq(21)$, to obtain

$$
\sum_{\substack{p a r(x y, u z)=1 \\ x y \times \text { odd } u z \\ D}} 1 \leq \sum_{\substack{p a r(x y, u z)=1 \\ x y \times \text { even } u z \\ D}} 1,
$$

and we proved the equivalence part of Theorem 1. Therefore, if (3) holds, then the number of edge pairs crossing odd times in $D$ is as small as possible. If $D$ is nice, then the number of crossings in $D, \operatorname{cr}(D)$, is equal to the number of non-adjacent edge pairs crossing odd times, $c r$-iodd $(D)$. Therefore

$$
C R-I O D D(G) \leq C R(G) \leq \operatorname{cr}(D)=c r-\operatorname{iodd}(D)=C R-I O D D(G),
$$


implying that $D$ is optimal drawing for $C R(G)$.

\section{References}

[1] M. R. Garey and D. S. Johnson, Crossing number is NP-complete, SIAM J. Alg. Discrete Methods 4 (1983), 312-316.

[2] R. K. Guy, The decline and fall of Zarankiewicz's theorem, in F. Harary (ed.), Proof Techniques in Graph Theory, Academic Press, New York, London, 1969, 63-69.

[3] J. Pach and G. Tóth, Which crossing number is it anyway? in: Proc. 39th Annual Symposium on Foundation of Computer Science, IEEE Press, Baltimore, 1998, 617-626; and J. Comb. Theory Ser. B 80 (2000), 225-246.

[4] M. J. Pelsmajer, M. Schaefer and Štefankovič, Odd crossing number is not crossing number, in: Graph Drawing, Lecture Notes in Computer Science 3843, Springer, Berlin, 2006, 386-396.

[5] L. A. Székely, A successful concept for measuring non-planarity of graphs: the crossing number, Discrete Math. 276 (2003), 1-3, 331-352.

[6] L. A. Székely, Zarankiewicz crossing number conjecture, in: M. Hazewinkel (ed.), Kluwer Encyclopaedia of Mathematics, Supplement III, Kluwer Academic Publishers, 2002, 451-452.

[7] P. Turán, A note of welcome, J. Graph Theory 1 (1977), 7-9.

[8] W. T. Tutte, Toward a theory of crossing numbers, J. Combinatorial Theory 8 (1970), 45-53.

[9] D. R. Woodall, Cyclic-order graphs and Zarankiewicz's crossing-number conjecture J. Graph Theory 17 (1993), 657-671.

[10] K. Zarankiewicz, On a problem of P. Turán concerning graphs, Fund. Math. 41 (1954), 137-145. 